\title{
REMARKS ON THE TIGHTNESS OF COCYCLES
}

\author{
BY \\ JON AARONSON (TEL AVIV) \\ AND BENJAMIN WEISS (JERUSALEM)
}

Dedicated to the memory of Anzelm Iwanik

\begin{abstract}
We prove a generalised tightness theorem for cocycles over an ergodic probability preserving transformation with values in Polish topological groups. We also show that subsequence tightness of cocycles over a mixing probability preserving transformation implies tightness. An example shows that this latter result may fail for cocycles over a mildly mixing probability preserving transformation.
\end{abstract}

Let $(\Omega, \mathcal{B}, m)$ be a probability space, let $T: \Omega \rightarrow \Omega$ be an ergodic probability preserving transformation, let $G$ be a Polish topological group and let $\phi: \Omega \rightarrow G$ be measurable.

We consider $S_{n}$, the random walk or cocycle on $G$ defined by

$$
S_{0}(\omega)=e, \quad S_{n+1}(\omega):=\phi\left(T^{n} \omega\right) S_{n}(\omega) .
$$

This random walk is generated by the skew product transformation $T_{\phi}$ : $X \times G \rightarrow X \times G$ where $T_{\phi}^{n}(\omega, y)=\left(T^{n} \omega, S_{n}(\omega) y\right)$. In case $G$ is a locally compact topological group, $T_{\phi}$ preserves the measure $m \times m_{G}$ where $m_{G}$ is a left Haar measure on $G$.

1. Tightness theorem. We consider the situation where $\left\{m\right.$-dist. $\left(S_{n}\right)$ : $n \geq 1\}$ is tight in the sense that for every $\varepsilon>0$, there is a compact $C \subset G$ such that $\sup _{n \geq 1} m\left(S_{n} \notin C\right)<\varepsilon$ (equivalently, tightness is precompactness in the space $\mathcal{P}(G)$ of probability measures on $G$ ). One way this can happen is when $\phi$ is cohomologous to a compact-group-valued function, i.e. there is a compact subgroup $K \subseteq G$ and measurable $\psi: \Omega \rightarrow K, g: \Omega \rightarrow G$ such that $\phi(\omega)=g(T \omega)^{-1} \psi(\omega) g(\omega)$; then $S_{n}(\omega)=g\left(T^{n} \omega\right)^{-1} k_{n}(\omega) g(\omega)$ where $k_{n}(\omega):=\psi\left(T^{n-1} \omega\right) \psi\left(T^{n-2} \omega\right) \ldots \psi(\omega) \in K$.

Tightness Theorem. The distributions $\left\{m\right.$-dist. $\left.\left(S_{n}\right): n \geq 1\right\}$ are tight in $\mathcal{P}(G) \Leftrightarrow \phi$ is cohomologous to a compact-group-valued function.

2000 Mathematics Subject Classification: Primary 28D05. 
Remarks about $\Leftarrow .1)$ The $\Leftarrow$ of the tightness theorem is an easy consequence of the tightness of a single probability on a Polish space (Prokhorov's theorem, see [Par]) and the probability preserving property of $T$.

2) If $m$ is not absolutely continuous with respect to some $T$-invariant probability on $(\Omega, \mathcal{B})$ then $\Leftarrow$ may fail.

In this case, there is a set $W \in \mathcal{B}$ with $m(W)>0$ and a sequence $n_{k} \rightarrow \infty$ such that $\left\{T^{-n_{k}} W: k \geq 1\right\}$ are disjoint (such a set is called weakly wandering). Given a noncompact Polish space $G$, we choose $x_{0} \in G$ and a sequence $y_{k} \in G, y_{k} \rightarrow \infty$ (i.e. for each compact $C \subset G, y_{k} \notin C$ eventually) and define $f: \Omega \rightarrow G$ by

$$
f(x)= \begin{cases}y_{k}, & x \in T^{-n_{k}} W(k \geq 1), \\ x_{0}, & x \in \Omega \backslash \bigcup_{k=1}^{\infty} T^{-n_{k}} W .\end{cases}
$$

It follows that $\left\{m\right.$-dist. $\left.\left(f \circ T^{n}\right): n \geq 1\right\}$ cannot be tight in $\mathcal{P}(G)$ since $m\left(\left[f \circ T^{n_{k}}=y_{k}\right]\right) \geq m(W) \nrightarrow 0$.

If $G$ is a noncompact Polish topological group, we set $\phi=f^{-1} f \circ T$ and obtain a coboundary for which the distributions $\left\{m\right.$-dist. $\left.\left(S_{n}\right): n \geq 1\right\}$ are not tight in $\mathcal{P}(G)$.

In case $G$ has no nontrivial compact subgroups, the tightness theorem boils down to the so-called coboundary theorem:

The distributions $\left\{m\right.$-dist. $\left.\left(S_{n}\right): n \geq 1\right\}$ are tight in $\mathcal{P}(G) \Leftrightarrow \phi$ is a coboundary.

The first version of the coboundary theorem seems to be:

$L^{2}$ Coboundary Theorem [Leo]. If $\left\{Z_{n}: n \geq 1\right\}$ is a wide sense stationary process, then there exists a wide sense stationary process $\left\{Y_{n}\right.$ : $n \geq 1\}$ such that $Z_{n}=Y_{n}-Y_{n+1}$ iff $\sup _{n \geq 1} \mathbb{E}\left(\left|\sum_{k=1}^{n} Z_{k}\right|^{2}\right)<\infty$.

Proof. If there is $\left\{Y_{n}: n \geq 1\right\}$ wide sense stationary such that $Z_{n}=$ $Y_{n}-Y_{n+1}$, then $\sum_{k=1}^{n} Z_{k}=Y_{1}-Y_{n+1}$ and $\left\|\sum_{k=1}^{n} Z_{k}\right\|_{2} \leq 2\left\|Y_{1}\right\|_{2}$ for all $n \geq 1$.

Conversely, if $\left\|\sum_{k=1}^{n} Z_{k}\right\|_{2} \leq M$ for all $n \geq 1$, then by weak* sequential compactness of norm bounded sets, there are $N_{a} \rightarrow \infty$ and a r.v. $Y=$ $Y\left(Z_{1}, Z_{2}, \ldots\right)$ such that

$$
\frac{1}{N_{a}} \sum_{n=1}^{N_{a}} \sum_{k=1}^{n} Z_{k} \rightarrow Y
$$

where $\rightarrow$ denotes weak convergence in $L^{2}$. Write $Y_{n}:=Y\left(Z_{n}, Z_{n+1}, \ldots\right)$. Then $\left\{Y_{n}: n \geq 1\right\}$ is a wide sense stationary process and

$$
\frac{1}{N_{a}} \sum_{n=1}^{N_{a}} \sum_{k=1}^{n} Z_{k+\nu-1} \rightarrow Y_{\nu} \quad \forall \nu \geq 1 .
$$


It follows that

$$
\begin{aligned}
Y_{\nu+1} & <\frac{1}{N_{a}} \sum_{n=1}^{N_{a}} \sum_{k=\nu+1}^{n+\nu} Z_{k}=\frac{1}{N_{a}} \sum_{n=1}^{N_{a}}\left(\sum_{k=\nu}^{n+\nu-1} Z_{k}+Z_{n+\nu}-Z_{\nu}\right) \\
& =\frac{1}{N_{a}} \sum_{n=1}^{N_{a}} \sum_{k=1}^{n} Z_{k+\nu-1}+\frac{1}{N_{a}} \sum_{n=1}^{N_{a}} Z_{n+\nu}-Z_{\nu} \rightarrow Y_{\nu}-Z_{\nu}
\end{aligned}
$$

because $\left\|\sum_{n=1}^{N_{a}} Z_{n+\nu}\right\|$ is uniformly bounded.

Leonov's theorem has the $L^{p}$ analogues:

$L^{p}$ Coboundary Theorem. Let $(X, \mathcal{B}, m, T)$ be a probability preserving transformation, let $1 \leq p<\infty$ and let $f: X \rightarrow \mathbb{R}$ be measurable. There exists $g \in L^{1}(m)$ such that $f=g-g \circ T$ iff $\sup _{n>1}\left\|\sum_{k=1}^{n} f \circ T^{k}\right\|_{p}<\infty$.

The proof of the $L^{p}$ coboundary theorem is the same as that of Leonov with Komlos type convergence replacing weak convergence when $p=1$.

The coboundary theorem is established in [Sch1] for the case $G=\mathbb{R}$, and in [Mo-Sch] for $G$ locally compact, second countable, Abelian without compact subgroups.

The tightness theorem for locally compact, second countable groups was established in [Sch2]; related partial results are given in [Co] and [Zim].

Bradley has proved $\Rightarrow$ of the coboundary theorem assuming only that $T$ is measurable: in [Br1] for $G=\mathbb{R}$, in [Br2] for $G$ a Banach space and in [Br3] for $G$ a group of upper triangular matrices.

The present methods can be stretched to prove the $\Rightarrow$ of the tightness theorem assuming only that $T$ is measurable and invertible.

BAsic Lemma. If the family $\left\{P\right.$-dist. $\left.\left(S_{n}\right): n \geq 1\right\}$ is tight in $\mathcal{P}(G)$, then there is a measurable $P: \Omega \rightarrow \mathcal{P}(G)$ such that

$$
P_{T \omega}(A)=P_{\omega}\left(\phi(\omega)^{-1} A\right) \quad(A \in \mathcal{B}(G)) .
$$

This basic lemma is implicit in $[\mathrm{Br} 1]$ for $G=\mathbb{R}$. The general proof is essentially as in [Br1] (see below).

The coboundary theorem for $\mathbb{R}$ is easily established using it ([Br1]). Indeed if for $\omega \in \Omega, \mu(\omega)$ is defined as the minimal number satisfying

$$
P_{\omega}((-\infty, \mu(\omega)]), P_{\omega}([\mu(\omega), \infty)) \geq 1 / 2,
$$

then $\mu: \Omega \rightarrow \mathbb{R}$ is measurable and (since $P_{T \omega}(A)=P_{\omega}(A-\phi(\omega))$ ) we have $\mu(T \omega)=\mu(\omega)-\phi(\omega)$.

The proof of the tightness theorem given the basic lemma uses a generalisation of the characterisation of invariant measures for group extensions in [Key-New]. The proof is an adaptation of Lemańczyk's proof of [Key-New] in [Lem]. See also the proof of Theorem 8.3.2 in [A]. 
Proof of the basic lemma. Choose first $K_{\nu} \subset K_{\nu+1} \subset \ldots \subset G$, a sequence of compact sets in $G$ with the property (ensured by tightness) that

$$
m\left(\left[S_{n} \in K_{\nu}^{\mathrm{c}}\right]\right) \leq 1 / 4^{\nu} \quad \forall n, \nu \geq 1 .
$$

Consider the random measures $W_{n}: \Omega \rightarrow \mathcal{P}(G)$ defined by

$$
W_{n}(A):=\frac{1}{n} \sum_{j=1}^{n} 1_{A}\left(S_{j}\right) .
$$

Next, for $\nu \geq 1$ let $\mathcal{A}_{\nu} \subset C\left(K_{\nu}\right)$ be a countable family, dense in $C\left(K_{\nu}\right)$; and let $\mathcal{A}=\bigcup_{\nu=1}^{\infty} \mathcal{A}_{\nu}$.

We now claim that there are $n_{k} \rightarrow \infty$ and $L: \mathcal{A} \rightarrow L^{\infty}(\Omega)$ such that

$$
\int_{G} f d W_{n_{k}} \rightarrow L(f) \quad \text { weak* in } L^{\infty}(\Omega) \forall f \in \mathcal{A} .
$$

This is shown using weak* precompactness of $L^{\infty}(\Omega)$-bounded sets, and a diagonalisation.

By possibly passing to a subsequence, we can ensure that for each $f \in \mathcal{A}$, there is $N_{f}$ such that

$$
\left|\int_{X}\left(\left(\int_{G} f d W_{n_{k}}-L(f)\right)\left(\int_{G} f d W_{n_{j}}-L(f)\right)\right) d m\right|<\frac{1}{2^{k}} \quad \forall k \geq N_{f}, j<k,
$$

whence $([\operatorname{Rev}])$

$$
\frac{1}{N} \sum_{k=1}^{N} \int_{G} f d W_{n_{k}} \rightarrow L(f) \quad \text { a.e. } \forall f \in \mathcal{A}
$$

and hence (by density) for all $f \in \bigcup_{\nu=1}^{\infty} C\left(K_{\nu}\right)$.

By the Chebyshev-Markov inequality,

$$
\begin{aligned}
m\left(L\left(1_{K_{\nu}^{\mathrm{c}}}\right)>1 / 2^{\nu}\right) \leftarrow m\left(W_{n_{k}}\left(K_{\nu}^{\mathrm{c}}\right)>1 / 2^{\nu}\right) & <2^{\nu} \int_{X} W_{n_{k}}\left(K_{\nu}^{\mathrm{c}}\right) d m \\
& <1 / 2^{\nu} \quad \forall \nu \geq 1
\end{aligned}
$$

and so by the Borel-Cantelli lemma, $L\left(1_{K_{\nu}^{\mathrm{c}}}\right) \leq 1 / 2^{\nu}$ a.e. for $\nu$ large.

It follows that there is a measurable $P: \Omega \rightarrow \mathcal{P}(G)$ such that $L(f)(\omega)=$ $\int_{G} f d P_{\omega}$ for all $f \in \mathcal{A}$.

To see that $P_{T \omega}=P_{\omega} \circ R_{\phi(\omega)}\left(R_{g}(y):=y g\right)$, note that

$$
\begin{aligned}
\int_{G} f d W_{n}(T \omega) & =\frac{1}{n} \sum_{j=1}^{n} f\left(S_{j}(T \omega)\right) \\
& =\frac{1}{n} \sum_{j=1}^{n} f\left(S_{j+1}(\omega) \phi(\omega)^{-1}\right)=\frac{1}{n} \sum_{j=2}^{n+1} f \circ R_{\phi(\omega)^{-1}}\left(S_{j}(\omega)\right)
\end{aligned}
$$




$$
\begin{aligned}
& =\int_{G} f \circ R_{\phi(\omega)^{-1}} d W_{n}(\omega) \pm \frac{2\|f\|_{\infty}}{n} \\
& =\int_{G} f d W_{n}(\omega) \circ R_{\phi(\omega)} \pm \frac{2\|f\|_{\infty}}{n} .
\end{aligned}
$$

Proof of $\Rightarrow$ in the tightness theorem. Given probabilities $\omega \mapsto p_{\omega}$ on $G$ satisfying

$$
p_{T \omega}=p_{\omega} \circ L_{\phi(\omega)^{-1}},
$$

define a probability $\mu \in \mathcal{P}(\Omega \times G)$ by

$$
\mu(A \times B):=\int_{A} p_{\omega}(B) d m(\omega) .
$$

We first note that this probability is $T_{\phi}$-invariant:

$$
\begin{aligned}
\int_{X \times G}(u \otimes v) \circ T_{\phi} d \mu & =\int_{X} u(T x) \int_{G} v(\phi(x) y) d p_{x}(y) d m(x) \\
& =\int_{X} u(T x) \int_{G} v(y) d p_{T x}(y) d m(x) \\
& =\int_{X} u(x) \int_{G} v(y) d p_{x}(y) d m(x)=\int_{X \times G} u \otimes v d \mu .
\end{aligned}
$$

Almost every ergodic component $P$ of $\mu$ has a disintegration over $m$ of the form

$$
P(A \times B):=\int_{A} \widetilde{p}_{\omega}(B) d m(\omega)
$$

where $\omega \mapsto \widetilde{p}_{\omega} \in \mathcal{P}(G)$ is measurable, and $\widetilde{p}_{T \omega}=\widetilde{p}_{\omega} \circ R_{\phi(\omega)}$. Fix one such $P$.

Define $p \in \mathcal{P}(G)$ by $p(B):=P(\Omega \times B)$. There are compact sets $C_{1} \subset$ $C_{2} \subset \ldots$ such that $\bigcup_{n=1}^{\infty} C_{n}=G \bmod p$. Define compact subsets $\left\{K_{n}\right.$ : $n \geq 0\}$ by

$$
K_{0}:=\{e\}, \quad K_{n+1}=\left(K_{n} \cup C_{n}\right)\left(K_{n} \cup C_{n}\right)^{-1}\left(K_{n} \cup C_{n}\right)\left(K_{n} \cup C_{n}\right)^{-1} .
$$

Evidently, $G_{0}:=\bigcup_{n=1}^{\infty} K_{n}$ is a subgroup of $G$ and $p\left(G \backslash G_{0}\right)=0$, whence $\tilde{p}_{\omega}\left(G \backslash G_{0}\right)=0$ for $m$-a.e. $\omega \in \Omega$.

Next, consider the space $C_{\mathrm{B}}\left(G_{0}\right)$ of bounded, continuous, $\mathbb{R}$-valued functions on $G_{0}$ (equipped with the supremum norm) and set

$$
\mathcal{C}:=\left\{f \in C_{\mathrm{B}}\left(G_{0}\right): \sup _{y \in K_{n}^{\mathrm{c}}}|f(y)| \underset{n \rightarrow \infty}{\longrightarrow} 0\right\} .
$$

Evidently $\mathcal{C}=\overline{\bigcup_{n=1}^{\infty} C_{\mathrm{B}}\left(K_{n}\right)}$ is separable, and $f \in \mathcal{C} \Rightarrow f \circ R_{g} \in \mathcal{C}$ for all $g \in G_{0}$ (since if $g \in K_{i}$, then $x \notin K_{n+i} \Rightarrow x g \notin K_{n}$ ).

For each $a \in G, P \circ Q_{a}$ (where $\left.Q_{a}(\omega, y):=(\omega, y a)\right)$ is also an ergodic $T_{\phi}$-invariant probability (since $T_{\phi} \circ Q_{a}=Q_{a} \circ T_{\phi}$ ), and therefore either 
$P \circ Q_{a}=P$ or $P \circ Q_{a} \perp P$. Define $H:=\left\{a \in G_{0}: P \circ Q_{a}=P\right\}$, a closed subgroup of $G_{0}$. For a.e. $\omega \in \Omega, p_{\omega}(A a)=p_{\omega}(A)(a \in H, A \in \mathcal{B}(G))$.

Consider the Banach space $\mathcal{M}\left(\Omega \times G_{0}\right)$ of bounded measurable functions $\Omega \times G_{0} \rightarrow \mathbb{R}$ equipped with the supremum norm. We need a separable subspace $\mathcal{A} \subset \mathcal{M}\left(\Omega \times G_{0}\right)$ which separates the points of $\Omega \times G_{0}$ such that $f \in \mathcal{A} \Rightarrow f \circ Q_{a} \in \mathcal{A}$ for all $a \in G_{0}$. In particular,

$$
a, b \in G_{0}, \int_{\Omega \times G} f d P \circ Q_{a}=\int_{\Omega \times G} f d P \circ Q_{b} \quad \forall f \in \mathcal{A} \Rightarrow P \circ Q_{a}=P \circ Q_{b} .
$$

To obtain such a subspace, fix a compact metric topology on $\Omega$ generating $\mathcal{B}$; then $\mathcal{A}=C(\Omega) \otimes \mathcal{C}$ is as needed.

By Birkhoff's ergodic theorem,

$$
\frac{1}{n} \sum_{k=0}^{n-1} f \circ T_{\phi}^{k}(\omega, y) \rightarrow \int_{\Omega \times G} f d P \quad \text { a.e. } \forall f \in L^{1}(P) .
$$

Set

$$
Y:=\left\{(\omega, y) \in \Omega \times G_{0}: \frac{1}{n} \sum_{k=0}^{n-1} f \circ T_{\phi}^{k}(\omega, y) \rightarrow \int_{\Omega \times G} f d P \forall f \in \mathcal{A}\right\} .
$$

Since $\mathcal{A}$ is a separable subspace of $\mathcal{M}\left(\Omega \times G_{0}\right)$, the set $Y$ is determined by a countable subcollection of $\mathcal{A}$, whence $Y \in \mathcal{B}\left(\Omega \times G_{0}\right)$, and by Birkhoff's ergodic theorem $P(Y)=1$. For $\omega \in \Omega$, set $Y_{\omega}=\left\{y \in G_{0}:(\omega, y) \in Y\right\}$. We claim that $Y_{\omega}$ is a coset of $H$ whenever it is nonempty.

To see this, suppose that $a \in G$. Then for all $f \in \mathcal{A}$ and for a.e. $(x, y)$ $\in Y$,

$$
\frac{1}{n} \sum_{k=0}^{n-1} f \circ T_{\phi}^{k}(\omega, y a) \rightarrow \int_{\Omega \times G} f \circ Q_{a} d P=\int_{\Omega \times G} f d P \circ Q_{a}^{-1} .
$$

Thus, $(\omega, y a) \in Y$ iff $P \circ Q_{a}^{-1}=P$, equivalently $a \in H$; and $Y_{\omega}$ is indeed a coset of $H$ whenever it is nonempty (i.e. a.e.).

By the analytic section theorem, there is a measurable $h: \Omega \rightarrow G$ such that $h(\omega) \in Y_{\omega}$ for a.e. $\omega \in \Omega$, whence $Y_{\omega}=h(\omega) H$.

Now let $P_{\omega}^{\prime} \in \mathcal{P}(G)$ be defined by $P_{\omega}^{\prime}(A):=p_{\omega}\left(h(\omega)^{-1} A\right)$. Clearly $P_{\omega}^{\prime}(H)=1$ and $P_{\omega}^{\prime}(A a)=P_{\omega}^{\prime}(A)(a \in H, A \in \mathcal{B}(G))$. Thus by [Weil], $H$ is compact and $P_{\omega}^{\prime}=m_{H}$, Haar measure on $H$.

Defining $\Psi: \Omega \times G \rightarrow \Omega \times G$ by $\Psi(\omega, y):=(\omega, h(\omega) y)$, we have $P \circ \Psi^{-1}=$ $m \times m_{H}$. If $V:=\Psi \circ T_{\phi} \circ \Psi^{-1}$ then $m \times m_{H} \circ V=m \times m_{H}$ and $V=T_{\psi}$ where $\psi(\omega):=h(\omega) \phi(\omega) h(\omega)^{-1}$.

Since $\left(\Omega \times G, \mathcal{B}(\Omega \times G), m \times m_{H}, V\right)$ is a probability preserving transformation, we see that $\psi: \Omega \rightarrow H$. 
2. Subsequence tightness. Let $(X, \mathcal{B}, m, T)$ be a mixing probability preserving transformation and let $\phi: X \rightarrow \mathbb{R}$ be measurable. Bradley [Br4] showed that if the stochastic process $\left\{\phi \circ T^{n}: n \geq 1\right\}$ is strongly Rosenblatt mixing, then either

1) $\sup _{r \in \mathbb{R}} m\left(\left[\left|S_{n}-r\right| \leq C\right]\right) \rightarrow 0$ for every $0<C<\infty$, or

2) there are constants $a_{n}$ such that $\left\{m\right.$-dist. $\left.\left(S_{n}-a_{n}\right): n \geq 1\right\}$ is tight (whence $\phi$ is cohomologous to a constant).

A weaker version of this generalises to an arbitrary stationary stochastic process driven by a mixing probability preserving transformation.

THEOREM 2. Suppose that $(X, \mathcal{B}, m, T)$ is a mixing probability preserving transformation and that $\phi: X \rightarrow \mathbb{R}$ is measurable. If there are $n_{k} \rightarrow \infty$ and $d_{k} \in \mathbb{R}$ such that $\left\{m\right.$-dist. $\left.\left(S_{n_{k}}-d_{k}\right): k \geq 1\right\}$ is tight, then there are $a \in \mathbb{R}$ and $g: \Omega \rightarrow \mathbb{R}$ measurable such that $\phi(\omega)=a+g(T \omega)-g(\omega)$. If $\sup _{k}\left|d_{k}\right|<\infty$, then $a=0$.

P r o of. Consider $(X \times X, \mathcal{B} \otimes \mathcal{B}, m \times m, T \times T)$, and $\phi, \phi^{\prime}: X \times X \rightarrow \mathbb{R}$ defined by $\phi(x, y):=\phi(x), \phi^{\prime}(x, y):=\phi(y)$.

- We first show that $\left\{m \times m\right.$-dist. $\left.\left(S_{n}-S_{n}^{\prime}\right): n \geq 1\right\}$ is tight. Let $\varepsilon>0$ and choose $M>0$ such that $m\left(\left[\left|S_{n_{k}}-d_{k}\right|>M / 2\right]\right)<\varepsilon / 2$ for all $k \geq 1$. By mixing of $T$, for all $n \geq 1$,

$$
m\left(\left[\left|S_{n}-S_{n} \circ T^{n_{k}}\right|>M\right]\right) \rightarrow m \times m\left(\left[\left|S_{n}-S_{n}^{\prime}\right|>M\right]\right)
$$

as $k \rightarrow \infty$. Now

$$
S_{n}-S_{n} \circ T^{n_{k}}=S_{n}-S_{n+n_{k}}+S_{n_{k}}=S_{n_{k}}-S_{n_{k}} \circ T^{n},
$$

whence

$$
\begin{aligned}
m\left(\left[\left|S_{n}-S_{n} \circ T^{n_{k}}\right|>M\right]\right) & =m\left(\left[\left|S_{n_{k}}-S_{n_{k}} \circ T^{n}\right|>M\right]\right) \\
& \leq 2 m\left(\left[\left|S_{n_{k}}-d_{k}\right|>M / 2\right]\right)<\varepsilon .
\end{aligned}
$$

- Next, as in [Br4], there are $a_{n} \in \mathbb{R}$ such that $\left\{m\right.$-dist. $\left.\left(S_{n}-a_{n}\right): n \geq 1\right\}$ is tight. To see this, given $\varepsilon>0$, let $M(\varepsilon)>0$ be such that

$$
m \times m\left(\left[\left|S_{n}-S_{n}^{\prime}\right|>M(\varepsilon)\right]\right)<\varepsilon^{2} \quad \forall n \geq 1 .
$$

It follows that

$$
\begin{aligned}
m\left(\left\{x \in X: m\left(\left[\mid S_{n}-\right.\right.\right.\right. & \left.\left.\left.\left.S_{n}(x) \mid>M(\varepsilon)\right]\right)>\varepsilon\right\}\right) \\
& \leq \frac{1}{\varepsilon} \int_{X} m\left(\left[\left|S_{n}-S_{n}(x)\right|>M(\varepsilon)\right]\right) d m(x) \\
& =\frac{1}{\varepsilon} m \times m\left(\left[\left|S_{n}-S_{n}^{\prime}\right|>M(\varepsilon)\right]\right)<\varepsilon \quad \forall n \geq 1,
\end{aligned}
$$


whence there are $a_{n}(\varepsilon) \in \mathbb{R}$ such that

$$
m\left(\left[\left|S_{n}-a_{n}(\varepsilon)\right|>M(\varepsilon)\right]\right) \leq \varepsilon \quad \forall n \geq 1 .
$$

Set $a_{n}=a_{n}(1 / 3)$. For each $0<\varepsilon<1 / 2, n \geq 1$, we have

$$
m\left(\left[\left|S_{n}-a_{n}(\varepsilon)\right|<M(\varepsilon)\right] \cap\left[\left|S_{n}-a_{n}\right|<M(1 / 3)\right]\right)>0,
$$

whence $\left|a_{n}-a_{n}(\varepsilon)\right|<M(1 / 3)+M(\varepsilon)$ and

$$
m\left(\left[\left|S_{n}-a_{n}\right|>2 M(\varepsilon)+M(1 / 3)\right]\right)<\varepsilon \quad \forall n \geq 1 .
$$

- We show that there is an $a \in \mathbb{R}$ such that $\sup _{n \geq 1}\left|a_{n}-n a\right|<\infty$. To this end, note that there is an $M>0$ such that

$$
\left|a_{k+l}-a_{k}-a_{l}\right|<M \quad \forall k, l \geq 1 .
$$

Indeed, if $m\left(\left[\left|S_{n}-a_{n}\right|>K\right]\right)<1 / 8$ for all $n \geq 1$, then (since $S_{k+l}=$ $\left.S_{k}+S_{l} \circ T^{k}\right)$

$$
\begin{aligned}
m\left(\left[\left|S_{k+l}-a_{k}-a_{l}\right|\right.\right. & >2 K]) \\
\leq & m\left(\left[\left|S_{k}-a_{k}\right|>K\right] \cup\left[\left|S_{l} \circ T^{k}-a_{l}\right|>K\right]\right)<1 / 4,
\end{aligned}
$$

whence

$$
m\left(\left[\left|S_{k+l}-a_{k}-a_{l}\right| \leq 2 K\right] \cap\left[\left|S_{k+l}-a_{k+l}\right| \leq K\right]\right)>0
$$

and $\left|a_{k+l}-a_{k}-a_{l}\right| \leq 3 K$ for $k, l \geq 1$.

By $(\ddagger)$, there are $N_{k} \rightarrow \infty$ and $b_{\nu} \in \mathbb{R}(\nu \geq 1)$ such that

$$
\frac{1}{N_{k}} \sum_{j=1}^{N_{k}}\left(a_{j+\nu}-a_{j}\right) \rightarrow b_{\nu} \quad \text { as } k \rightarrow \infty \forall \nu \geq 1 .
$$

It follows from $(\ddagger)$ that

$$
\left|b_{\nu}-a_{\nu}\right|=\lim _{k \rightarrow \infty}\left|\frac{1}{N_{k}} \sum_{j=1}^{N_{k}}\left(a_{j+\nu}-a_{j}-a_{\nu}\right)\right| \leq M
$$

and that

$$
\begin{aligned}
b_{\nu+\mu} & \leftarrow \frac{1}{N_{k}} \sum_{j=1}^{N_{k}}\left(a_{j+\mu+\nu}-a_{j}\right) \\
& =\frac{1}{N_{k}} \sum_{j=1}^{N_{k}}\left(a_{j+\mu}-a_{j}\right)+\frac{1}{N_{k}} \sum_{j=\mu+1}^{N_{k}+\mu}\left(a_{j+\nu}-a_{j}\right) \\
& =\frac{1}{N_{k}} \sum_{j=1}^{N_{k}}\left(a_{j+\mu}-a_{j}\right)+\frac{1}{N_{k}} \sum_{j=1}^{N_{k}}\left(a_{j+\mu}-a_{j}\right) \pm \frac{M+\left|a_{\mu}\right|}{N_{k}} \\
& \rightarrow b_{\mu}+b_{\nu} .
\end{aligned}
$$

Thus $b_{\nu}=\nu a$ and $\left|a_{\nu}-\nu a\right| \leq M$ where $a=b_{1}=\lim _{n \rightarrow \infty} a_{n} / n$. 
In case $\sup _{k}\left|d_{k}\right|<\infty$, because of the tightness of $\left\{m\right.$-dist. $\left.\left(S_{n_{k}}\right): k \geq 1\right\}$ we have $\sup _{k>1}\left|a_{n_{k}}\right|<\infty$, whence $a=0$.

- It now follows from the coboundary theorem that $\phi$ is cohomologous to $a$.

3. An example. We show that there is a probability preserving transformation $(X, \mathcal{B}, m, T)$ which is mildly mixing in the sense that there is no $A \in \mathcal{B}$ with $0<m(A)<1$ such that $\liminf _{n \rightarrow \infty} m\left(A \triangle T^{n} A\right)=0$ (see $\S 2.7$ of $[\mathrm{A}]$ ), but there is a measurable function $\phi: X \rightarrow \mathbb{R}$ such that $T_{\phi}$ is ergodic and for some $n_{k} \rightarrow \infty, \limsup _{k \rightarrow \infty}\left|S_{n_{k}}\right|<\infty m$-almost everywhere.

Chacon's transformation [Cha]. This transformation $(X, \mathcal{B}, m, T)$ is defined inductively on $X:=\bigcup_{n=1}^{\infty} C_{n} \subset \mathbb{R}$ where $m=$ Lebesgue measure.

Here $C_{n}=\bigcup_{k=0}^{l_{n}-1} T^{k} J_{n}$ where

- $l_{1}=1, l_{n+1}=3 l_{n}+1\left(\Rightarrow l_{n}=\left(3^{n}-1\right) / 2\right)$;

- $\left\{T^{k} J_{n}: 0 \leq k \leq l_{n}-1\right\}$ are disjoint intervals of length $1 / 3^{n-1}$ and $T: T^{k} J_{n} \rightarrow T^{k+1} J_{n}$ is a translation;

- $C_{n+1}$ is obtained by writing $J_{n}=\bigcup_{i=0}^{2} J_{n, i}$ where the $J_{n, i}(i=0,1,2)$ are disjoint intervals of length $1 / 3^{n}$ and setting $J_{n+1}:=J_{n, 0}$ and

$$
T^{k} J_{n+1}:= \begin{cases}T^{k} J_{n, 0}, & 0 \leq k \leq l_{n}-1, \\ T^{k-l_{n}} J_{n, 1}, & l_{n} \leq k \leq 2 l_{n}-1, \\ \mathcal{S}_{n+1}, & k=2 l_{n}, \\ T^{k-2 l_{n}-1} J_{n, 2}, & 2 l_{n}+1 \leq k \leq 3 l_{n}=l_{n+1}-1\end{cases}
$$

where $\mathcal{S}_{n+1}$ is an interval of length $1 / 3^{n}$, disjoint from $C_{n}$ (called the spacer).

The set $X$ has finite measure which can be normalized to equal one but we keep the standard Lebesgue measure in order to simplify the later formulae. We first give a proof of the ergodicity based on a careful analysis of how the intervals $T^{k} J_{n}$ approximate arbitrary measurable sets. This analysis will also be the base for our proof of the mild mixing property.

Define

$$
\mathcal{C}_{n}:=\left\{U_{n}(K):=\bigcup_{k \in K} T^{k} J_{n}: K \subset\left\{0,1, \ldots, l_{n}-1\right\}\right\} .
$$

For $A \in \mathcal{B}, \varepsilon>0$ and $n \geq 1$ define

$$
K_{A, \varepsilon}^{(n)}:=\left\{0 \leq k \leq l_{n}-1: m\left(T^{k} J_{n} \cap A\right)<\varepsilon m\left(J_{n}\right)\right\} \subset\left\{0,1, \ldots, l_{n}-1\right\} .
$$

Evidently, for $A, B \in \mathcal{B}$ disjoint and $0<\varepsilon<1 / 2, K_{A, \varepsilon}^{(n)}$ and $K_{B, \varepsilon}^{(n)}$ are disjoint.

It is standard that for all $A \in \mathcal{B}$ and $\varepsilon>0$, there is $N_{A, \varepsilon}$ such that

$$
\left|E_{A}^{(n)}\right|<\varepsilon l_{n} \quad \forall n \geq N_{A, \varepsilon}
$$

where

$$
E_{A}^{(n)}:=\left\{0,1, \ldots, l_{n}-1\right\} \backslash\left(K_{A, \varepsilon}^{(n)} \cup K_{A^{c}, \varepsilon}^{(n)}\right),
$$


whence (for such $n$ )

$$
m\left(U_{n}\left(K_{A, \varepsilon}^{(n)}\right) \backslash A\right)=\sum_{k \in K_{A, \varepsilon}^{(n)}} m\left(T^{k} J_{n} \backslash A\right)<\varepsilon m\left(C_{n}\right)
$$

and

$$
\begin{aligned}
m\left(A \backslash U_{n}\left(K_{A, \varepsilon}^{(n)}\right)\right) & =m\left(A \cap U_{n}\left(K_{A^{\mathrm{c}}, \varepsilon}^{(n)}\right)\right)+m\left(A \cap U_{n}\left(E_{A}^{(n)}\right)\right) \\
& \leq \sum_{k \in K_{A^{\mathrm{c}}, \varepsilon}^{(n)}} m\left(T^{k} J_{n} \backslash A\right)+\varepsilon m\left(C_{n}\right)<2 \varepsilon m\left(C_{n}\right)
\end{aligned}
$$

and $m\left(A \triangle U_{n}\left(K_{A, \varepsilon}^{(n)}\right)\right)<3 \varepsilon m\left(C_{n}\right)$. Henceforth, we let $n_{A, \varepsilon}$ be the minimal $N$ with $\left|E_{A}^{(n)}\right|<\varepsilon l_{n}$ for all $n \geq N$.

Conversely, suppose that $A \in \mathcal{B}$ and $U=U_{n}(K) \in \mathcal{C}_{n}$ satisfy $m(A \triangle U)<$ $\varepsilon m(U)$. Then

$$
\begin{aligned}
\sum_{k \in K, m\left(T^{k} J_{n} \backslash A\right) \geq \sqrt{\varepsilon} m\left(J_{n}\right)} m\left(T^{k} J_{n}\right) & \\
& \leq \frac{1}{\sqrt{\varepsilon}} \sum_{k \in K, m\left(T^{k} J_{n} \backslash A\right) \geq \sqrt{\varepsilon} m\left(J_{n}\right)} m\left(T^{k} J_{n} \backslash A\right) \\
& \leq \frac{1}{\sqrt{\varepsilon}} m(U \backslash A)<\sqrt{\varepsilon}
\end{aligned}
$$

and

$$
\begin{aligned}
\sum_{k \in K^{\mathrm{c}}, m\left(T^{k} J_{n} \backslash A^{\mathrm{c}}\right) \geq \sqrt{\varepsilon} m\left(J_{n}\right)} m\left(T^{k} J_{n}\right) \\
\leq \frac{1}{\sqrt{\varepsilon}} \sum_{k \in K^{\mathrm{c}}, m\left(T^{k} J_{n} \backslash A^{\mathrm{c}}\right) \geq \sqrt{\varepsilon} m\left(J_{n}\right)} m\left(T^{k} J_{n} \backslash A^{\mathrm{c}}\right) \\
\leq \frac{1}{\sqrt{\varepsilon}} m(A \backslash U)<\sqrt{\varepsilon}
\end{aligned}
$$

whence

$$
\left|K \backslash K_{A, \varepsilon}^{(n)}\right|,\left|K^{\mathrm{c}} \backslash K_{A^{\mathrm{c}}, \varepsilon}^{(n)}\right| \leq \sqrt{\varepsilon} l_{n}
$$

and $n \geq n_{A, 2 \sqrt{\varepsilon}}$.

To see (the well known fact $[\mathrm{Fr}])$ that $(X, \mathcal{B}, m, T)$ is an ergodic measure preserving transformation, let $A \in \mathcal{B}$ with $m(A)>0$ satisfy $T A=A$. Evidently, $K_{A}^{(n)} \neq \emptyset \Rightarrow K_{A}^{(n)}=\left\{0,1, \ldots, l_{n}-1\right\}$, whence $U_{n}\left(K_{A, \varepsilon}^{(n)}\right)=C_{n}$.

It follows that $m(A)>m\left(C_{n}\right)(1-3 \varepsilon)$ for all $\varepsilon>0$ and $n \geq n_{A, \varepsilon}$, whence $A=X \bmod m$.

In [Cha] it was shown that Chacon's transformation $(X, \mathcal{B}, m, T)$ is weakly mixing and not strongly mixing. We next claim that it is mildly mixing. For a related result, see $[\mathrm{F}-\mathrm{K}]$. 
To see this, we first need some notation to record how sets in $\mathcal{C}_{n}$ appear in $C_{n+2}$. Define $e_{j}(0 \leq j \leq 7)$ by

$$
e_{j}:= \begin{cases}0, & j=0,2,3,6, \\ 1, & j=1,4,5,7,\end{cases}
$$

$\kappa_{j}=\kappa_{j, n}$ by

$$
\kappa_{0}=0, \quad \kappa_{j+1}:=\kappa_{j}+l_{n}+e_{j}
$$

and

$$
X_{j}=X_{j, n}:=\bigcup_{i=0}^{l_{n}-1} T^{i+\kappa_{j, n}} J_{n+2} \quad(0 \leq j \leq 8) .
$$

Then given $n \geq 1, K \subset\left\{0,1, \ldots, l_{n}-1\right\}$ and $U=U_{n}(K) \in \mathcal{C}_{n}$, we have

$$
T^{\kappa_{j, n}}\left(U \cap X_{0}\right)=\bigcup_{i \in K} T^{i+\kappa_{j, n}} J_{n+2}=U \cap X_{j} \quad(0 \leq j \leq 7)
$$

and

$$
T^{l_{n}+e_{j}}\left(U \cap X_{j}\right)=U \cap X_{j+1} .
$$

Next suppose that $A \in \mathcal{B}, \varepsilon>0$ and $n \geq n_{A, \varepsilon}$. Then

$$
m\left(T^{i+\kappa_{j, n}} J_{n+2} \cap A\right)<9 \varepsilon m\left(J_{n+2}\right) \quad \forall i \in K_{A^{\mathrm{c}}, \varepsilon}^{(n)}, 0 \leq j \leq 8
$$

and

$$
m\left(T^{i+\kappa_{j, n}} J_{n+2} \backslash A\right)<9 \varepsilon m\left(J_{n+2}\right) \quad \forall i \in K_{A, \varepsilon}^{(n)}, 0 \leq j \leq 8 ;
$$

whence

$$
m\left(T^{\kappa_{j, n}}\left(A \cap X_{0}\right) \triangle\left(A \cap X_{j}\right)\right)<36 \varepsilon .
$$

Now suppose $A \in \mathcal{B}$ with $m(A)>0$ satisfies $\liminf _{n \rightarrow \infty} m\left(A \triangle T^{n} A\right)=0$. We claim that $A=T^{-1} A$.

To see this, fix $\varepsilon>0$. Then there are $n \geq n_{A, \varepsilon}$ and $N \in\left[l_{n}, l_{n+1}-1\right]$ such that $m\left(A \triangle T^{N} A\right)<\varepsilon$, whence there is $B \in \mathcal{C}_{n}$ such that $m\left(B \triangle T^{N} B\right)<3 \varepsilon$. Write $N=a l_{n}+b$ where $a=1,2$ and $0 \leq b \leq l_{n}$. For $0 \leq j \leq 6-a$ we have

$$
T^{N} X_{j}=T^{a l_{n}+b} X_{j}=T^{b-e_{j, a}} X_{j+a}
$$

where $e_{j, 1}=e_{j}$ and $e_{j, 2}=e_{j}+e_{j+1}$. Thus, on the one hand

$$
\begin{aligned}
T^{N}\left(B \cap X_{j}\right) & =T^{N} B \cap T^{N} X_{j} \\
& \approx^{3 \varepsilon} B \cap T^{N} X_{j}=B \cap T^{b-e_{j, a}} X_{j+a} \quad(0 \leq j \leq 7)
\end{aligned}
$$

(where $C \approx^{\eta} D$ means $m(C \triangle D)<\eta$ ) and on the other hand

$$
T^{N}\left(B \cap X_{j}\right)=T^{b-e_{j, a}}\left(B \cap X_{j+a}\right) \quad(0 \leq j \leq 6-a)
$$

whence

$$
\begin{aligned}
B \cap X_{j+a} & \approx^{3 \varepsilon} T^{-b+e_{j, a}} B \cap X_{j+a} & & \forall 0 \leq j \leq 6-a, \\
B & \approx^{27 \varepsilon} T^{-b+e_{j, a}} B & & \forall 0 \leq j \leq 6-a,
\end{aligned}
$$


whence (choosing $j, j^{\prime}$ with $e_{j, a}-e_{j^{\prime}, a}=1$ )

$$
B \approx^{54 \varepsilon} T B \Rightarrow A \approx^{56 \varepsilon} T A \text {. }
$$

The cocycle. This cocycle $\phi: X \rightarrow \mathbb{Z}$ will be defined successively as a sum of coboundaries. Define $g^{(n)}: C_{n+2} \rightarrow \mathbb{Z}$ by

$$
g^{(n)}(x)= \begin{cases}1, & x \in \mathcal{S}_{n+1}, \\ -3, & x \in \mathcal{S}_{n+2}, \\ 0, & \text { else. }\end{cases}
$$

Note that

(‡) $\quad \forall n \geq 1, k \geq n+2, \quad T^{N} X_{i, k}=X_{i+j, k} \Rightarrow g_{N}^{(n)} \equiv 0$ on $X_{i, k}$

(this is because $\left.g_{N}^{(n)}\right|_{X_{i, k}}=\left.j g_{l_{k}}^{(n)}\right|_{J_{k}}=0$ ); whereas for all $U \in \mathcal{C}_{n}$,

$$
U \cap T^{-\left(2 l_{n}+1\right)} U \cap\left[g_{2 l_{n}+1}^{(n)}=1\right] \supset U \cap \bigcup_{k=0,1,3,7} X_{k, n}=: U \cap Y_{n},
$$

whence

$$
m\left(U \cap T^{-\left(2 l_{n}+1\right)} U \cap\left[g_{2 l_{n}+1}^{(n)}=1\right]\right) \geq \frac{4}{9} m(U) .
$$

Now fix a sequence $n_{k} \nearrow \infty$ such that

- $n_{k+1}>n_{k}+2$,

- $\sum_{j \geq k+1} m\left(\mathcal{S}_{n_{j}}\right)<m\left(J_{n_{k}}\right) /\left(45\left(2 l_{n_{k}}+1\right)\right)$

and define $\phi:=\sum_{k=1}^{\infty} g^{\left(n_{k}\right)}$.

Ergodicity of $T_{\phi}$. We see by $(\ddagger)$ that for all $k \geq 1$,

$$
\phi_{2 l_{n_{k}}+1}=\sum_{j \geq k} g_{2 l_{n_{k}}+1}^{\left(n_{j}\right)} \quad \text { on } Y_{n_{k}}
$$

whence

$$
\begin{aligned}
m\left(Y_{n_{k}} \cap\left[\phi_{2 l_{n_{k}}+1} \neq g_{2 l_{n_{k}}+1}^{\left(n_{k}\right)}\right]\right) & \leq \sum_{j \geq k+1} m\left(\left[g_{2 l_{n_{k}}+1}^{\left(n_{j}\right)} \neq 0\right]\right) \\
& \leq\left(2 l_{n_{k}}+1\right) \sum_{j \geq k+1} m\left(\mathcal{S}_{n_{j}}\right) \leq \frac{m\left(J_{n_{k}}\right)}{45}
\end{aligned}
$$

and for $U \in \mathcal{C}_{n_{k}}, U \neq \emptyset$, we have

$$
\begin{aligned}
m(U & \left.\cap T^{-\left(2 l_{n_{k}}+1\right)} U \cap\left[\phi_{2 l_{n_{k}}+1}=1\right]\right) \\
& \geq m\left(U \cap T^{-\left(2 l_{n_{k}}+1\right)} U \cap\left[g_{2 l_{n_{k}}+1}^{\left(n_{k}\right)}=1\right]\right)-m\left(\left[\phi_{2 l_{n_{k}}+1} \neq g_{2 l_{n_{k}}+1}^{\left(n_{k}\right)}\right]\right) \\
& \geq \frac{4}{9} m(U)-\frac{m\left(J_{n_{k}}\right)}{45} \geq \frac{19 m(U)}{45} .
\end{aligned}
$$


To show that $T_{\phi}: X \times \mathbb{Z} \rightarrow X \times \mathbb{Z}$ is ergodic, it suffices by [Sch1] to show that if $A \in \mathcal{B}, m(A)>0$ and $k \geq 1$ is large enough, then

$$
m\left(A \cap T^{-\left(2 l_{n_{k}}+1\right)} A \cap\left[\phi_{2 l_{n_{k}}+1}=1\right]\right)>0 .
$$

To see this, note that for $k \geq 1$ large enough, there exists $U \in \mathcal{C}_{n}$ with $m(A \triangle U)<2 m(U) / 45$, whence

$$
\begin{aligned}
m\left(A \cap T^{-\left(2 l_{n_{k}}+1\right)}\right. & \left.A \cap\left[\phi_{2 l_{n_{k}}+1}=1\right]\right) \\
& \geq m\left(U \cap T^{-\left(2 l_{n_{k}}+1\right)} U \cap\left[\phi_{2 l_{n_{k}}+1}=1\right]\right)-2 m(A \Delta U) \\
& \geq m(U) / 3>0 .
\end{aligned}
$$

Tightness of $\left\{m\right.$-dist. $\left.\left(S_{l_{n_{k}}}\right): k \geq 1\right\}$. We first claim that

$(\diamond)$

$$
\left|\left(\sum_{k=1}^{K} g^{\left(n_{k}\right)}\right)_{l_{N}}\right| \leq 3 \quad \forall K \geq 1, N \geq n_{K}+2 .
$$

To see this, we consider the tower $C_{N+2}$ which consists of $C_{N}$-blocks, and the spacers $\mathcal{S}_{N+1} \cup \mathcal{S}_{N+2}$ on which $\sum_{k=1}^{K} g^{\left(n_{k}\right)} \equiv 0$. The cocycle sum over a $C_{N}$-block is zero by construction.

An arbitrary cocycle sum of length $l_{N}$ in $C_{N+2}$ begins in the middle of a $C_{N}$-block, either passes over a spacer interval (in $\mathcal{S}_{N+1} \cup \mathcal{S}_{N+2}$ ) or not, and continues to the middle of the next $C_{N}$-block. In the second case, the cocycle sum will be as over a $C_{N}$-block, and will be zero. In the first case, it will be as over a $C_{N}$-block less one interval (the one before the starting place) and

$$
\left(\sum_{k=1}^{K} g^{\left(n_{k}\right)}\right)_{l_{N}}=-\sum_{k=1}^{K} g^{\left(n_{k}\right)}\left(x_{0}\right) .
$$

The claim $(\diamond)$ follows since $\sum_{k=1}^{K} g^{\left(n_{k}\right)}=0,1,-3$.

To prove our tightness claim, we prove that $m\left(\left[\left|S_{l_{n_{K}}}\right| \geq 4\right]\right) \rightarrow 0$ as $K \rightarrow \infty$. Indeed, by $(\diamond)$,

$$
\begin{aligned}
m\left(\left[\left|S_{l_{n_{K}}}\right| \geq 4\right]\right) & \leq m\left(\left[S_{l_{n_{K}}} \neq\left(\sum_{k=1}^{K} g^{\left(n_{k}\right)}\right)_{l_{n_{K}}}\right]\right) \\
& =m\left(\left[\left(\sum_{k=K+1}^{\infty} g^{\left(n_{k}\right)}\right)_{l_{n_{K}}} \neq 0\right]\right) \\
& \leq l_{n_{K}} m\left(\left[\sum_{k=K+1}^{\infty} g^{\left(n_{k}\right)} \neq 0\right]\right) \\
& \leq l_{n_{K}} \sum_{k=K+1}^{\infty} m\left(\mathcal{S}_{n_{k}}\right) \leq \frac{m\left(J_{n_{K}}\right)}{90} .
\end{aligned}
$$




\section{REFERENCES}

[A] J. Aaronson, An Introduction to Infinite Ergodic Theory, Math. Surveys Monogr. 50, Amer. Math. Soc., Providence, RI, 1997.

[Br1] R. C. Bradley, On a theorem of K. Schmidt, Statist. Probab. Lett. 23 (1995), 9-12.

[Br2] -, A "coboundary" theorem for sums of random variables taking values in a Banach space, Pacific J. Math. 178 (1997), 201-224.

[Br3] - A "multiplicative coboundary" theorem for some sequences of random matrices, J. Theoret. Probab. 9 (1996), 659-678.

$[\mathrm{Br} 4]-$, On the dissipation of the partial sums of a stationary strongly mixing sequence, Stochastic Process. Appl. 54 (1994), 281-290.

[Cha] R. V. Chacon, Weakly mixing transformations which are not strongly mixing, Proc. Amer. Math. Soc. 22 (1969), 559-562.

[Co] J.-P. Conze, Transformations cylindriques et mesures finies invariantes, Ann. Sci. Univ. Clermont Math. 17 (1979), 25-31.

[Fr] N. Friedman, Introduction to Ergodic Theory, van Nostrand, New York, 1970.

[F-K] N. Friedman and J. King, Rank one lightly mixing, Israel J. Math. 73 (1991), 281-288

[Key-New] H. Keynes and D. Newton, The structure of ergodic measures for compact group extensions, Israel J. Math. 18 (1974), 363-389.

[Lem] M. Lemańczyk, Ergodic compact Abelian group extensions, habilitation thesis, Nicholas Copernicus Univ., Toruń, 1990.

[Leo] V. P. Leonov, On the dispersion of time averages of a stationary random process, Theory Probab. Appl. 6 (1961), 93-101.

[Mo-Sch] C. C. Moore and K. Schmidt, Coboundaries and homomorphisms for nonsingular actions and a problem of $H$. Helson, Proc. London Math. Soc. 40 (1980), 443-475.

[Par] K. R. Parthasarathy, Introduction to Probability and Measure, Springer, New York, 1978.

[Rev] P. Revesz, On a problem of Steinhaus, Acta Math. Acad. Sci. Hungar. 16 (1965), 311-318.

[Sch1] K. Schmidt, Cocycles of Ergodic Transformation Groups, Lecture Notes in Math. 1, MacMillan of India, 1977.

[Sch2] —, Amenability, Kazhdan's property T, strong ergodicity and invariant means for ergodic group-actions, Ergodic Theory Dynam. Systems 1 (1981), 223236.

[Weil] A. Weil, L'intégration dans les groupes topologiques et ses applications, Actualités Sci. Indust. 869, Hermann, Paris, 1940.

[Zim] R. J. Zimmer, On the cohomology of ergodic group actions, Israel J. Math. 35 (1980), 289-300.

School of Mathematical Sciences

Tel Aviv University

69978 Tel Aviv, Israel

E-mail: aaro@math.tau.ac.il
Mathematical Institute

Hebrew University

Jerusalem, Israel

E-mail: weiss@math.huji.ac.il 